Disclosure of Interest: None declared

DOI: 10.1136/annrheumdis-2018-eular.5230

\section{AB0707 1 FOUR DISTINCT CLINICAL PHENOTYPES OF VASCULITIS AFFECTING MEDIUM-SIZED ARTERIES}

T. Shirai, H. Fujii, Y. Shirota, T. Ishii, H. Harigae. Hematology and Rheumatology, Tohoku University Graduate School of Medicine, Sendai, Japan

Background: Polyarteritis nodosa (PAN) is a necrotizing arteritis of mediumsized arteries. PAN is divided into systemic and cutaneous PAN (cPAN). cPAN is further classified into mild cPAN or severe cPAN, which presents with ulcer, necrosis, or neuritis. However, it is sometimes difficult to distinguish between severe cPAN and systemic PAN, and their optimal managements are still unclear. The aim of this study is to evaluate clinical characteristics of patients with necrotizing arteritis of medium and small artery.

Objectives: To evaluate the clinical characteristics of patients with necrotizing arteritis of medium-sized arteries in order to further clarify the subtypes of PAN. Methods: Forty-nine patients diagnosed with necrotizing arteritis of mediumsized arteries between 2008 and 2017 at our institution were enrolled to this study Patients with evidence of glomerulonephritis or vasculitis in arterioles, capillaries, or venules, which are hallmarks of small-vessel vasculitis, were excluded. Clinical backgrounds, laboratory findings including inflammatory markers and antineutrophil cytoplasmic antibodies (ANCA), affected organs, treatments, and rates of relapse and death were evaluated.

Results: Among 49 patients, 11 patients (22\%) presented with systemic vasculitis. Organ involvement was diverse and ranged from central nervous system to cutaneous manifestations. The remaining 38 patients were diagnosed as cPAN and further classified as mild CPAN (16 patients) or severe CPAN (22 patients: ulcer type, 9; neuritis type, 9; both, 4). There was one case of cPAN which resulted in renal damage. The clinical characteristics of mild cPAN included female predominance (87.5\%) and younger age (median, 32), and patients tend to have normal inflammatory markers and laboratory findings. Those of systemic PAN included older age (median, 69), higher levels of inflammatory markers, lower levels of serum proteins, and organ damage. Nonspecific elevation of autoantibodies is frequently observed. Particulary, $54.5 \%$ of systemic type possessed MPOANCA though the titers were significantly lower than those of microscopic polyangiitis, suggesting non-specific elevation of MPO-ANCA in Japanese population. Severe cPAN manifested with intermediate phenotypes, and inflammatory activities were significantly correlated with age $(p<0.001)$. Although the mortality rates were indistinguishable, the relapse rates of cPAN (ulcer type) were significantly higher than those of other types (88.9\%, Figure). The mean doses of prednisolone (PSL) used to treat mild cPAN, severe cPAN, and systemic type were 18.5, 38.6, and $39.1 \mathrm{mg} /$ day, respectively. Immunosuppressants were used in $20 \%$ of mild cPAN, $90.9 \%$ of severe cPAN, and $72.7 \%$ of systemic PAN patients. Most patients with cPAN (ulcer type) were initially treated by corticosteroid monotherapy, and immunosuppressive agents were added when they relapsed. Considering its high relapse rate, it might be reasonable to select combination therapy with cyclophosphamide for severe cPAN (ulcer type) as is proposed in nonsystemic vasculitic neuropathy.

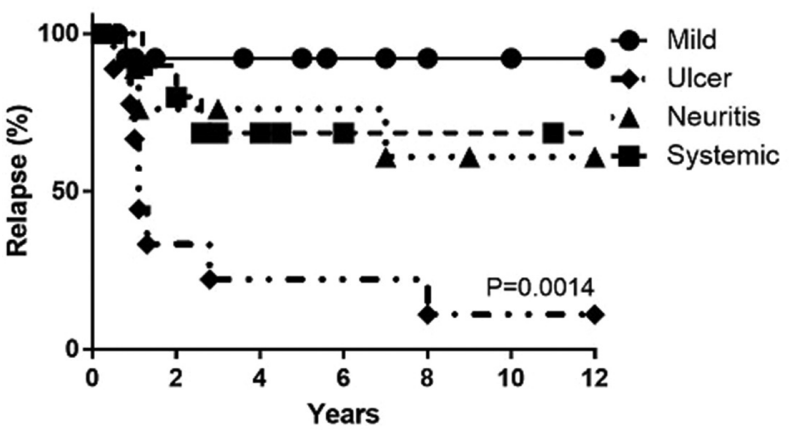

$\begin{array}{cccccccc}\text { Number at risk } & 0 y & 2 y & 4 y & 6 y & 8 y & 10 y & 12 y \\ \text { Mild } & 16 & 15 & 11 & 9 & 7 & 4 & 2 \\ \text { Ulcer } & 9 & 3 & 2 & 2 & 1 & 1 & 1 \\ \text { Neuritis } & 9 & 7 & 5 & 5 & 2 & 1 & 1 \\ \text { Systemic } & 11 & 9 & 7 & 5 & 2 & 2 & 1\end{array}$

Abstract AB0707 - Figure 1
Conclusions: The clinical characteristics of mild cPAN, severe cPAN (ulcer type), severe cPAN (neuritis type), and systemic PAN were distinct from each other. Particularly, patients with severe cPAN (ulcer type) had higher relapse rates, thus indicating the importance of combination therapy in this patient cohort. Disclosure of Interest: None declared

DOI: 10.1136/annrheumdis-2018-eular.2247

\section{AB0708 DIAGNOSIS AND MANAGEMENT OF PATIENTS WITH GIANT CELL ARTERITIS (GCA)- A RE-AUDIT IN RHEUMATOLOGY IN THE BELFAST HEALTH AND SOCIAL CARE TRUST (BHSCT)}

\section{U.A. Laverty, E. Banks, M. McHenry. NHS, Belfast, Ireland}

Background: Patients should meet at least 3/5 American College of Rheumatology (ACR) criteria for a diagnosis of GCA. British Society of Rheumatology (BSR) guidelines on the management of GCA from 2010 emphasise the importance of early referral for specialist management. TAB should be considered depending on local experience and availability of other imaging modalities. Biopsy should be at least $10 \mathrm{~mm}$ in length and ideally done within 14 days of presentation. TABs performed in BHSCT over a three-year period were audited in 2014.

Objectives: We re-audited the Rheumatology TAB referrals in the BHSCT from August 2016 to August 2017 to assess how biopsies influenced the management of patients with GCA in comparison to the previous audit.

Methods: Histopathology based LabCentre search detected 36 TABs within the given period. A retrospective audit was carried out with the use of Electronic Care Record and 16 of these patients were Rheumatology based TAB referrals. A proforma was used to aid data collection.

Results: Female:Male ratio was 2.2:1, age range was 61-91 with a mean age of 76. $94 \%$ of patients presented with headache. Of those patients referred for biopsy, $100 \%$ already fulfilled 2 of ACR criteria, compared with only $80 \%$ in previous audit. TAB was positive in $25 \%$. In those with a positive biopsy, $75 \%$ had an ESR $>50$. All patients were on steroid treatment at the time of biopsy. In $42 \%$ of patients with a negative biopsy, steroids were rapidly reduced. $100 \%$ of patients with a positive biopsy continued on steroids. $68 \%$ of biopsies were greater than $10 \mathrm{~mm}$ compared with $38 \%$ in the previous audit. $68 \%$ of patients had a TAB within 14 days of commencing on steroids, with $31 \%$ having biopsy within 7 days of commencing steroids. $63 \%$ of patients developed steroid related complications. In $56 \%$ of patients the biopsy result changed patient management.

Conclusions: The percentage of appropriate referrals for biopsy (based on the ACR criteria) has improved compared with the previous audit. The length of biopsy improved. $32 \%$ of patients were waiting longer than 2 weeks for biopsy but some of these patients presented late to the rheumatology team. Biopsy results changed management in $56 \%$ of patients. Areas for consideration include improvement in time to biopsy and biopsy length.

\section{REFERENCE:}

[1] BSR and BHPR guidelines for management of giant cell arteritis. Bhaskar Dasgupta, et al. Rheumatology, Volume 49, Issue 8, 1 August 2010 Pages 1594-1597. Published: 05 April 2010

Disclosure of Interest: None declared

DOI: 10.1136/annrheumdis-2018-eular.7339

\section{AB0709 1 SWITCHING FROM ORIGINATOR INFLIXIMAB TO BIOSIMILAR INFLIXIMAB: EFFICACY AND SAFETY IN A COHORT OF PATIENTS WITH ESTABLISHED BEHÇET'S DISEASE}

V. Venerito $^{1}$, G. Lopalco ${ }^{1}$, L. Cantarini ${ }^{2}$, C. Fabiani ${ }^{3}$, M. Nivuori ${ }^{1}$, F. Cacciapaglia ${ }^{1}$, M. Galeazzi ${ }^{2}$, G. Lapadula', F. lannone ${ }^{1} .{ }^{1}$ Department of Emergency and Organ Transplantations, Rheumatology Unit, University of Bari "Aldo Moro", Bari; ${ }^{2}$ Department of Medical Sciences, Surgery and Neurosciences, Rheumatology Unit, Policlinico "Le Scotte", University of Siena, Siena; ${ }^{3}$ Department of Ophthalmology, Humanitas Clinical and Research Center, Rozzano, Italy

Background: Infliximab (IFX) has been proved to be effective in several organ involvement of Behçet's Disease (BD). A recent report ${ }^{1}$ describing rapid loss of efficacy of biosimilar IFX after switching from originator IFX suggests the necessity to exercise caution regarding the automatic substitution of originator IFX with biosimilar IFX in patients achieving remission with originator IFX.

Objectives: The purpose of the present study was to describe our experience with biosimilar IFX CT-P13 in patients affected with BD, who were switched from originator IFX. 
Methods: Retrieved data including demographic characteristics, clinical manifestations and previous treatments were collected. All patients met the ISG and/or ICBD classification criteria for Behçet's Disease. In order to evaluate disease activity, the BD Current Activity Form (BDCAF) has been evaluated before starting biosimilar, at three, six and nine months after switching to CT-P13. The occurrence of adverse events was also recorded. Wilcoxon matched-pairs signedranks test was carried out to evaluate differences between BDCAF distributions pre-switch and either at three, at six and at nine months after switching.

Results: Thirteen caucasian adult BD patients (mean age $39.77 \pm 7.46$ years) with a mean disease duration of $12.54 \pm 4.21$ years, underwent IFX treatment at licensed dosage for a period of $117.66 \pm 48.01$ months. After $106.92 \pm 46.37$ months of treatment with originator IFX, all of them were switched to CT-P13 biosimilar IFX. At 3 months after switching, none of them had discontinued CT-P13 biosimilar IFX treatment. No significant difference was noticed between BDCAF mean score assessed at switch and 3 months after switching $(p=0.15)$. At 6 months follow up, 2/13 patients (15.38\%) discontinued CT-P13 biosimilar IFX treatment, both for recurrence of mucocutaneous involvement. One out of 2 patients who discontinued CT-P13 IFX had previously experienced a disease flare under originator IFX therapy, requiring a modification of ongoing therapy. BDCAF mean score assessed before and 6 months after switching were not significantly different $(p=0.81)$. Nine months after switching 2 out of the remaining 11 patients were lost at follow up. Once more, no difference was shown between BDCAF mean score assessed at switch and at 9 months follow up $(p=0.85)$. No adverse events occurred during the observed period.

\begin{tabular}{lc}
\hline Female $\mathrm{n}(\%)$ & $3(23.08 \%)$ \\
\hline Age at Onset (mean \pm SD) & $27.15 \pm 10.02$ \\
Clinical Manifestations $\mathrm{n}(\%)$ & \\
Uveitis & $10(76.92)$ \\
Oral Aphtosis & $9(69.23)$ \\
Genital Aphtosis & $7(53.85)$ \\
Cutaneous Involvement & $7(53.85)$ \\
Concomitant Treatment $\mathrm{n}(\%)^{*}$ & \\
Colchicine & $5(38.46)$ \\
csDMARDs & $4(30.77)$ \\
Corticosteroids & $1(7.69)$ \\
\hline
\end{tabular}



Abstract AB0709 - Figure 1

Conclusions: Despite the short follow up period, these data suggest that switching BD patients from originator IFX to CT-P13 seems to be effective and safe; only a small percentage of patients experienced relapse of symptoms, whereas a significant modification of BDCAF pre-switch and post-switch was not noticed. Although encouraging, these results need to be confirmed over a longer follow up period and on larger cohorts of patients.

\section{REFERENCE:}

[1] Cantini F, et al. (2017) Rapid loss of efficacy of biosimilar infliximab in three patients with Behcet's disease after switching from infliximab originator. Eur J Rheumatol 4 (4):288-290

Disclosure of Interest: None declared

DOI: 10.1136/annrheumdis-2018-eular.3093

\section{$\mathrm{AB} 0710$ \\ INTERFERON A2A FOR THE TREATMENT OF REFRACTORY BEHCWET'S DISEASE UVEITIS}

W. Zheng ${ }^{1}$, J. Shi ${ }^{1}$, C. Zhao ${ }^{2}$, J. Liu ${ }^{1}$, J. Zhou ${ }^{1}$, F. Gao ${ }^{2}$, M. Zhang ${ }^{2}$.

${ }^{1}$ Rheumatology and Clinical Immunology, ${ }^{2}$ Ophthalmology, Peking Union Medical College Hospital, BEIJING, China

Background: Behçet's Disease uveitis(BDU) mostly involved bilateral panuveitis and retinal vasculitis, which are very challenging to treat. Interferon alfa-2a (IFNo $2 \mathrm{a})$ has been shown to have comparable effectiveness and tolerance profiles for BDU as tumour necrosis factor (TNF) inhibitors in a number of studies with a much lower cost. IFN $\alpha-2 a$ treatment combined with corticosteroids without immunosuppressants was common in previous studies. We herein report a cohort of highly refractory BDU patients who experienced recurrence despite aggressive treatment with multiple immunosuppressants at their therapeutic doses.

Objectives: To investigate the efficacy and safety of IFN $\alpha 2 a$ treatment in combination with corticosteroids and immunosuppressants in patients with refractory BDU.

Methods: Clinical records of refractory BDU patients who underwent IFN $\alpha 2 a$ treatment in our centre between 2015 and 2017 were retrospectively reviewed IFN $\alpha 2$ a was initially given 3.0 million IU (MIU) subcutaneously daily for 4 weeks, on the basis of conventional corticosteroid and immunosuppressive therapy. The dosage was gradually tapered down to $3.0 \mathrm{MIU}$ three times or even once per week for maintenance. Primary outcome measure was success rate and changes in ocular relapse rates before and after initiation of IFN- $\alpha 2 a$ treatment. Disease activity, corticosteroid- and immunosuppressive agent-sparing effects and potential side effects were considered to be secondary outcomes.

Results: A total of 26 patients (23 males and 3 females) were included, with a median disease course of 41 months (range 5-168) before IFN $\alpha 2$ a treatment. No major organ involvement except for ocular inflammation was noted. Concomitant medical conditions include chronic hepatitis B virus infection in 2 patients, pulmo nary tuberculosis in 1 patient who was treated with antitubercular agents in the meanwhile. Prior to IFN $\alpha 2 a$ therapy, the median minimum dosage of corticosteroids was 20 (range 15-45) mg/day prednisone or equivalent, and 17 patients $(65.4 \%)$ were treated with at least two immunosuppressive agents. Four received short terms of TNF $\alpha$ inhibitor therapy but stopped due to economic burden. Severe side effects related to previous therapies including femoral head necrosis and secondary hypertension were observed in some patients. Treatment success of IFN $\alpha 2 a$ was achieved in the majority of the patients $(24 / 26,92.3 \%)$. During a mean follow-up of IFN $\alpha 2 a$ therapy for $13.6 \pm 6.0$ months, the median rate of uveitis relapse decreased notably from 8 per patient-year (range 2-12) to 0 per patientyear (range $0-6)(p=0.000008)$. Oral corticosteroids were successfully decreased in 20 cases $(76.9 \%)$ and completely discontinued in 2 patients $(7.7 \%)$, with the median minimum dosage reduced from $20 \mathrm{mg} /$ day (range 15-45) to $15 \mathrm{mg} /$ day (range $0-50)(p=0.006221)$. Moreover, immunosuppressive agents were cut down on types and dosage in $15(57.7 \%)$ and 23 patients $(88.5 \%)$, respectively, and were totally quitted in 5 cases $(19.2 \%)$. Slight elevated liver and renal function parameters were detected in one and two patients, respectively. No other severe adverse events occurred. The serum autoantibodies were all negative during treatment with IFN $\alpha 2 \mathrm{a}$.

Conclusions: IFN $\alpha 2 a$, in combination with corticosteroids and immunosuppressants, was effective and relatively safe in refractory BDU, with a potential steroidand immunosuppressive agent-sparing effect.

Disclosure of Interest: None declared

DOI: 10.1136/annrheumdis-2018-eular.3453

\section{AB0711 TOCILIZUMAB FOR SEVERE/REFRACTORY VASCULAR BEHÇET'S DISEASE}

W. Zheng ${ }^{1}$, Y. Ding ${ }^{2}$, J. Liu ${ }^{1}$, J. Shi', C. Li ${ }^{1}$, Y. Zhao ${ }^{1}$, X. Zeng ${ }^{1} .{ }^{1}$ Rheumatology and Clinical Immunology, Peking Union Medical College Hospital, BEIJING;

${ }^{2}$ Rheumatology, The First Affiliated Hospital of Zhengzhou University, Zheng Zhou, China

Background: Vascular Behçet's Disease (BD) is a common yet severe complication of BD. Despite aggressive conventional therapy, vascular BD remains as one of the leading causes of morbidity of BD. Tocilizumab (TCZ), an IL-6 receptor antagonist, is an emerging biological agents for neurologic $\mathrm{BD}$ or ocular $\mathrm{BD}$, however, the efficacy of TCZ for vascular BD is unknown.

Objectives: To elucidate the efficacy and safety of TCZ for severe/refractory vas cular BD.

Methods: We retrospectively analysed the clinical data of vascular BD patients treated with TCZ in our centre between 2014 and 2017

Results: Seven patients (6 males and 1 female) were enrolled, with a mean age and median course of $32.9 \pm 9.1$ years old and 72 months (range 54 to 138), respectively. Multiple arterial lesions were documented in all patients, including arterial aneurysm $(n=5)$, stenosis $(n=4)$, occlusion $(n=3)$, and multiple venous thrombosis were documented in two patients. Recurrent aneurysms together with 
graft occlusion or endoleak occurred in two out of 3 aneurysms patients who underwent endovascular stent placements. As for concomitant medical conditions, one patient complicated with hepatitis B infection, and three complicated with latent tuberculosis infection and received anti-tuberculosis prophylaxis. Prior to TCZ therapy, all patients experienced disease progression despite high-dose glucocorticosteroid in combination with multiple immunosuppressants. All patients received TCZ, $8 \mathrm{mg} / \mathrm{kg}$ iv every 4 weeks for a median of 7 (range 3 to 15) doses, and baseline medium-to-low dose corticosteroid and immunosuppressants, Afte a median follow-up of 16 months (range 4 to 29), all patients achieved both clinical and serological improvements. Radiologic improvement of artery stenosis was demonstrated in one patient. The condition of one patient with endoleak after the stent placement remained stable without surgical intervention. No newly-onset arterial lesions or venous thrombosis were observed. The median ESR (18 vs $7 \mathrm{~mm} / \mathrm{h}, \mathrm{p}<0.05)$ and hsCRP level (30.6 vs $2.0 \mathrm{mg} / \mathrm{L}, \mathrm{p}<0.05)$ were also significantly decreased. Furthermore, corticosteroids dose was tapered from 27.1 $\pm 6.3 \mathrm{mg}$ to $8.9 \pm 1.2 \mathrm{mg}(\mathrm{p}<0.05)$, and immunosuppressants were tapered in number and dosage in $3(42.9 \%)$ and 3 patients (42.9\%), respectively. No serious adverse event or TB reactivation was observed.

Conclusions: TCZ, in combination with corticosteroids and immunosuppressants, was effective and well tolerated in severe/refractory vascular BD, with a favourable steroid- and immunosuppressant-sparing effect.

Disclosure of Interest: None declared

DOI: 10.1136/annrheumdis-2018-eular.4152

\section{AB0712 ELIGIBILITY OF PATIENTS WITH GIANT CELL ARTERITIS FOR ENTRY INTO A PROSPECTIVE RANDOMISED CONTROLLED TRIAL: A SINGLE-CENTRE EXPERIENCE}

\author{
W.A. Schmidt ${ }^{1}$, K. Hofheinz ${ }^{1}$, S. Burger ${ }^{1}$, V.S. Schäfer ${ }^{1,2}$, A. Juche ${ }^{1} .{ }^{1}$ Medical \\ Center for Rheumatology Berlin-Buch, IMMANUEL KRANKENHAUS BERLIN, \\ Berlin; ${ }^{2}$ Medizinische Klinik III, University of Bonn, Bonn, Germany
}

Background: The interest in pharmaceutical trials in giant cell arteritis (GCA) is increasing. Trial recruitment may be more challenging in GCA than in other rheumatic diseases because of a higher proportion of elderly patients who are additionally faced with a new diagnosis when being considered for trial participation Objectives: To analyse the eligibility of newly diagnosed and flaring GCA patients in one centre for randomization in the SIRRESTA trial (ClinicalTrials.gov Identifier, NCT02531633; sirukumab versus placebo), to list reasons for non-randomization, and to compare eligible with non-eligible patients.

Methods: All patients with newly diagnosed or relapsing GCA considered for trial participation between August 2016 until trial termination in October 2017 were included. The trial was prematurely terminated by the sponsor based on the decision to discontinue development of sirukumab in autoimmune diseases. Analysis of variance, two-sided Fisher's exact and Pearson's chi-squared tests were applied for calculating statistical significance.

Abstract AB0712 - Table 1. Comparison of eligible and non-eligible GCA patients for trial participation

\begin{tabular}{|c|c|c|c|c|}
\hline & Total & $\begin{array}{l}\text { Eligible for } \\
\text { trial }\end{array}$ & $\begin{array}{l}\text { Not eligible for } \\
\text { trial }\end{array}$ & Significance \\
\hline $\mathrm{N}$ & 95 & $12(13 \%)$ & $83(87 \%)$ & \\
\hline Age (SD) & $\begin{array}{l}71.1(8.3) \\
\text { years }\end{array}$ & $\begin{array}{c}65.3(8.0) \\
\text { years }\end{array}$ & $71.8(8.5)$ years & $0.008^{*}$ \\
\hline Females & $61 \%$ & $75 \%$ & $59 \%$ & 0.356 \\
\hline New diagnosis & $81 \%$ & $58 \%$ & $84 \%$ & $0.047^{\star}$ \\
\hline Cranial GCA only & $46 \%$ & $58 \%$ & $45 \%$ & 0.537 \\
\hline $\begin{array}{l}\text { Cranial and extracranial } \\
\text { GCA }\end{array}$ & $35 \%$ & $25 \%$ & $36 \%$ & 0.533 \\
\hline Extracranial GCA only & $19 \%$ & $17 \%$ & $19 \%$ & 1.0 \\
\hline Ischaemic events & $14 \%$ & $0 \%$ & $16 \%$ & 0.208 \\
\hline Headache & $59 \%$ & $58 \%$ & $59 \%$ & 1.0 \\
\hline Jaw claudication & $34 \%$ & $33 \%$ & $34 \%$ & 1.0 \\
\hline PMR & $62 \%$ & $83 \%$ & $59 \%$ & 0.125 \\
\hline ESR (SD) & $71(33) \mathrm{mm} / \mathrm{h}$ & $85(31) \mathrm{mm} / \mathrm{h}$ & $69(34) \mathrm{mm} / \mathrm{h}$ & 0.125 \\
\hline CRP (SD) & $90(69) \mathrm{mg} / \mathrm{l}$ & $121(83) \mathrm{mg} / \mathrm{l}$ & $85(65) \mathrm{mg} / \mathrm{l}$ & 0.095 \\
\hline
\end{tabular}

${ }^{*} \mathrm{p}<0,05$, significant; CRP, C-reactive protein; ESR, erythrocyte sedimentation rate; GCA giant cell arteritis; PMR, polymyalgia rheumatica; SD, standard deviation

Results: Ninety-five patients were pre-screened. Fifteen of these patients were screened and only 12 of the 95 (13\%) pre-screened GCA patients were eligible for randomization based on inclusion and exclusion criteria. The other 83 patients were not eligible for one or more of the following reasons: Concomitant diseases $(42 \%)$ including second autoimmune disease $(11 \%)$, malignancy $(6 \%)$, history of diverticulitis $(5 \%)$, hepatopathy $(2 \%)$, renal insufficiency $(1 \%)$ frailty $(6 \%)$ and dementia $(2 \%)$, ischaemic disease $(2 \% ; 1$ myocardial infarction and 1 severe tongue necrosis), abnormal or inconclusive tests for tuberculosis (5\%) and hepatitis (1\%); failure to meet inclusion criteria (41\%), including symptoms $(12 \%)$, histology/imaging (1\%), exceedance of maximum glucocorticoid dose $(7 \%)$, too low ESR and CRP (10\%), too long treatment duration (11\%); declined consent $(29 \%)$, before (11\%) and after receiving the consent form (18\%); too long distance to study centre $(5 \%)$.

Patients eligible for the trial were younger. They had no ischaemic complications (see table 1). A higher proportion of relapsing GCA patients were included. This subgroup of a larger trial represents only data from one centre. It may not be representative for the findings in other centres or the whole study.

Conclusions: Many newly diagnosed or relapsing GCA patients were not eligible for a trial due to concomitant diseases, failing inclusion criteria or declined consent. Eligible patients were younger; and more had relapsing disease.

Disclosure of Interest: W. Schmidt Grant/research support from: Roche, GSK (principle investigator), Consultant for: Roche, GSK, Sanofi, Speakers bureau: Roche, K. Hofheinz: None declared, S. Burger Grant/research support from: GSK (sub-investigator), V. Schäfer Grant/research support from: GSK (sub-investigator), A. Juche Grant/research support from: GSK (sub-investigator)

DOI: 10.1136/annrheumdis-2018-eular.425

\section{AB0713 $1 N T E R F E R O N-A L P H A$ FOR THE MANAGEMENT OF LOWER EXTREMITY DEEP VEIN THROMBOSIS IN BEHCET'S SYNDROME: A CASE SERIES}

Y. Ozguler, G. Hatemi, F. Cetinkaya, K. Tascilar, S. Ugurlu, E. Seyahi, H. Yazici, M. Melikoglu. Istanbul University Cerrahpasa Medical Faculty, ISTANBUL, Turkey

Background: Lower extremity deep vein thrombosis (LEDVT) is a disabling complication of Behçet's syndrome (BS). Relapses are frequent and cause permanent disability due to post-thrombotic syndrome. ${ }^{1}$ The management of LEDVT in Behcet's syndrome (BS) constitutes mainly of azathioprine (AZA) and corticosteroids (CS) as first-line agents. ${ }^{2}$ Interferon- $\alpha$ (IFN) has been used with good results in the management of eye involvement of BS. However data regarding its efficacy for vascular involvement has been scarse. ${ }^{3}$

Objectives: We aimed to evaluate the efficacy and safety of IFN for LEDVT in BS

Methods: All BS pts who had a first episode of acute LEDVT since March 2010 are being prospectively followed with a standard protocol in our dedicated BS centre. Acute LEDVT is confirmed by Doppler ultrasonography (DUS) at initial diagnosis and serial DUS assessment is performed and also repeated in case of clinical suspicion of relapse. Our standard treatment strategy consists of AZA and CSs as first-line treatment in pts with LEDVT. IFN has been used in pts who were refractory or intolerant to this regimen, or who had co-existing eye involvement. Our endpoints for assessing efficacy of IFN have been recanalisation of the index thrombus and prevention of relapses. Presence of recanalisation of thrombosed vein and extension of thrombosis have been assessed at each visit. Recanalisation has been assessed in the transverse plane and defined as the ratio of the vein area at maximum compressibility to the non-compressed vein area. Good recanalisation was defined as a ratio of at least $50 \%$. Adverse events during IFN use were recorded.

Results: 33 pts with LEDVT (26 M/7 F) were prospectively followed for a mean of 40.7 \pm 13.4 mo. Among these IFN was started in 18/33. In 2 pts IFN was started at the first episode of LEDVT due to co-existing uveitis. Seven pts were treated with IFN due to LEDVT relapses under AZA. In the remaining 9 pts, the reasons for switching from AZA to IFN were adverse events with AZA $(n=2)$, relapse of superficial thrombophlebitis $(n=4)$, leg ulcers due to severe post-thrombotic syndrome $(n=2)$ and eye involvement $(n=1)$. Among 17 pts treated with IFN mainly for vascular involvement during a mean follow-up of $29 \pm 20$ mo, 3 pts already had good recanalisation when starting IFN. In the remaining 14 pts, $13(93 \%)$ had good recanalisation under IFN. Two pts (11\%) experienced relapses. One of the 2 pts who had a relapse had had poor recanalisation despite IFN. In contrast, among the 29 pts treated with AZA, only $13(45 \%)$ had good recanalisation and $13(45 \%)$ pts experienced relapses. Nine fo the 13 pts who had relapses under AZA had poor recanalisation. Overall we observed 23 LEDVT relapses in 15 pts. Relapse rates were $29 \%, 37 \%$ and $45 \%$ at 6,12 and 24 months respectively. The only adverse event with interferon-alpha causing drug withdrawal was thyroiditis in 1 patient.

Conclusions: Relapse rate for LEDVT in BS is high despite AZA treatment. IFN seems to be a promising agent for preventing LEDVT relapses and achieving good recanalisation, an important predictor of relapse. The small number of pts and the lack of a parallel control group are the limitations of this prospective study.

REFERENCES:

[1] Melikoglu M. Arthritis Rheumatol 2014

[2] Alibaz-Oner F. Medicine (Baltimore) 2015

[3] Calguneri M. Ann Rheum Dis. 2003

Disclosure of Interest: None declared

DOI: 10.1136/annrheumdis-2018-eular.7197 